\title{
Acúfeno como síntoma inicial de plasmocitoma de hueso temporal
}

\author{
Tinnitus as the initial symptom of temporal bone plasmacytoma
}

Eugenia Hernández-Castillo ${ }^{1}$,Erika Celis-Aguilar ${ }^{1}$, Carlos Ochoa-Miranda ${ }^{1}$, César García-Valle

\section{Resumen}

Los tumores de base de cráneo son inusuales, entre ellos el plasmocitoma surge de las células plasmáticas y puede ser el precursor del mieloma múltiple. Existen 2 tipos de plasmocitoma: el óseo solitario y el extramedular. Además, el plasmocitoma temporal es aún menos frecuente. El acúfeno puede ser la presentación inicial de estos tumores inusuales. Se presenta caso de una paciente femenina de 74 años, que inicia con acúfeno derecho, así como hipoacusia derecha. Acompañado de dolor cervical y limitación de la flexión cervical e inestabilidad. Como único dato a la otoscopia derecha presenta hipervascularidad del promontorio. Se realizan estudios de imagen reportando tumor con erosión ósea de ambas porciones petrosas de hueso temporal, esfenoides y columna cervical. Se realizó una biopsia transesfenoidal reportando plasmocitoma CD 138 positivo. Se inició quimioterapia y radioterapia. El acúfeno unilateral debe estudiarse ampliamente ya que puede ser síntoma de diagnósticos inusuales como neoplasias de base de cráneo.

Palabras clave: Plasmocitoma, hueso temporal, acúfeno, radioterapia.

\begin{abstract}
Skull-base tumors are unusual, Plasmacytoma arises from plasma cells and could be the precursor of multiple myeloma. There are 2 types of plasmacytoma: solitary bone and extramedullary. Temporal bone plasmacytoma is even more infrequent. Tinnitus could be the initial symptom of this unusual tumors. We present the case of a 74-year-old female patient, who started with right tinnitus as well as right hearing loss. Accompanied by cervical pain and limitation of cervical flexion and instability. As the only data on right otoscopy, there was an hypervascular promontory. Imaging studies were performed reporting tumor with bone erosion of both petrous portions of temporal bone, sphenoid, and cervical spine. A transsphenoidal biopsy was performed, reporting positive CD 138 plasmacytoma. Chemotherapy and radiotherapy were indicated. Unilateral tinnitus should be fully studied because it might be a symptom of unusual diagnoses such as skull base neoplasms.
\end{abstract}

Keywords: Plasmacytoma, temporal bone, tinnitus, radiotherapy.
${ }^{1}$ Universidad Autónoma de Sinaloa, Centro de Investigación y Docencia en Ciencias de la Salud. Sinaloa, México.

Los autores declaran no tener conflictos de interés. Este trabajo fue presentado en modalidad de póster en el SMORL 69. Mazatlán, México, 2019.

Recibido el 2 de julio de 2020 Aceptado el 7 de octubre de 2020.

Correspondencia: Érika Celis-Aguilar Eustaquio Buelna \#91 Col. Gabriel Leyva, C.P. 80030 , Culiacán. Sinaloa, México. Email: erikacelis@hotmail.com

\section{Introducción}

Los tumores de base del cráneo son inusuales y los síntomas son inespecíficos o inexistentes hasta que crecen e invaden diversas estructuras. Es habitual que los pacientes presenten desequilibrio, acúfeno pulsátil unilateral, hipoacusia asimétrica leve, cefalea, espasmos o entumecimiento facial.

El plasmocitoma es una patología rara que pertenece al grupo de neoplasias malignas de células plasmáticas. Corresponde alrededor del $4 \%$ de todos los tumores de las células plasmáticas ${ }^{1}$, y se puede considerar como la lesión precursora del mieloma múltiple ${ }^{2}$, con una tasa de diseminación de mieloma múltiple que oscila entre el $8 \%$ y el $50 \%$. Dependiendo del origen de las células neoplásicas, los plasmocitomas solitarios se dividen en plasmocitoma óseo solitario y plasmocitoma extramedular. El primero debe su origen a la médula ósea y provoca osteólisis, mientras que el segundo se 
deriva de la capa submucosa de los tejidos y no afecta hueso.

El plasmocitoma extramedular es mucho más raro que el plasmocitoma óseo solitario, pero en la región de cabeza y cuello se presenta en más del $80 \%$ de los casos (tejido submucoso de vías respiratorias), lo que representa al menos el 1\% de las neoplasias malignas que se forman en la región de cabeza y cuello ${ }^{1}$, aunque tiene el doble de posibilidades de formarse en la columna vertebral que en cualquier otro hueso $^{3}$. La edad más común en la que se presenta el plasmocitoma extramedular es después de los 50 años y tiene más predilección por los hombres que por las mujeres en una proporción de $3-4: 1^{1}$. El plasmocitoma del hueso temporal es una patología aún más inusual ${ }^{1}$.

En cuanto a la presentación clínica, el acúfeno ha sido poco descrito en el plasmocitoma de hueso temporal, asimismo, existen otros síntomas reportados en la literatura como son la plenitud ótica, hipoacusia, diplopía, otalgia y cefalea ${ }^{1-3}$. El tratamiento de elección para el plasmocitoma extramedular aún no se encuentra bien definido debido a la poca frecuencia con la que este tumor se presenta; aunque existen distintas opciones de tratamiento, lo más utilizado es quimioterapia y radioterapia. El acúfeno puede ser un síntoma inicial en el plasmocitoma de base de cráneo, el objetivo de este caso clínico es enfatizar en la presentación otológica.

\section{Caso Clínico}

Paciente femenino de 74 años con antecedentes de importancia; hija con cáncer de mama, tabaquismo positivo durante 30 años a razón de 3 cajetillas al día. Inicia padecimiento actual un mes previo a la consulta presentando acúfeno derecho molesto e incapacitante de tono medio, de aparición gradual, no pulsátil e hipoacusia derecha gradual. El acúfeno era intermitente y le impedía hacer actividades diarias, así como la concentración. La paciente refería que debido al acúfeno unilateral había consultado numerosos facultativos previamente. Además, presentaba dolor cervical y limitación de la flexión cervical, relacionado con pérdida del plano de sustentación un día previo.

A la exploración física, pupilas centrales, normorreflécticas, isocóricas, movimientos oculares sin limitaciones. Otoscopia derecha: conducto auditivo permeable, membrana derecha íntegra, con hipervascularidad en el promontorio derecho; otoscopia izquierda: membrana timpánica izquierda íntegra, aperlada, referencias conservadas. Rinoscopia con cornetes en ciclo. Weber lateraliza a la derecha, Rinne positivo bilateral. Se realizan estudios de imagen reportando tumor con erosión ósea de clivus, esfenoides y seno cavernoso, con erosión a ambos ápex petrosos de predominio derecho, así como extensión a C1, C2, C3 (Figuras 1, 2 y 3 ).

Se realizó una biopsia transesfenoidal nasal con diagnóstico de plasmocitoma CD 138 positivo. La paciente presentó parálisis del sexto par derecho transitorio posquirúrgico, debido al empaquetamiento del esfenoides con material hemostático durante la cirugía. Se inicia quimioterapia y radioterapia. Después de 2 sesiones, no toleró por toxicidad la quimioterapia. Continúa en seguimiento en la consulta externa y terapia física.

Figura 1. Tomografía de cráneo simple en ventana para hueso corte sagital con erosión ósea de clivus, C1, C2, C3, ocupación de seno esfenoidal.

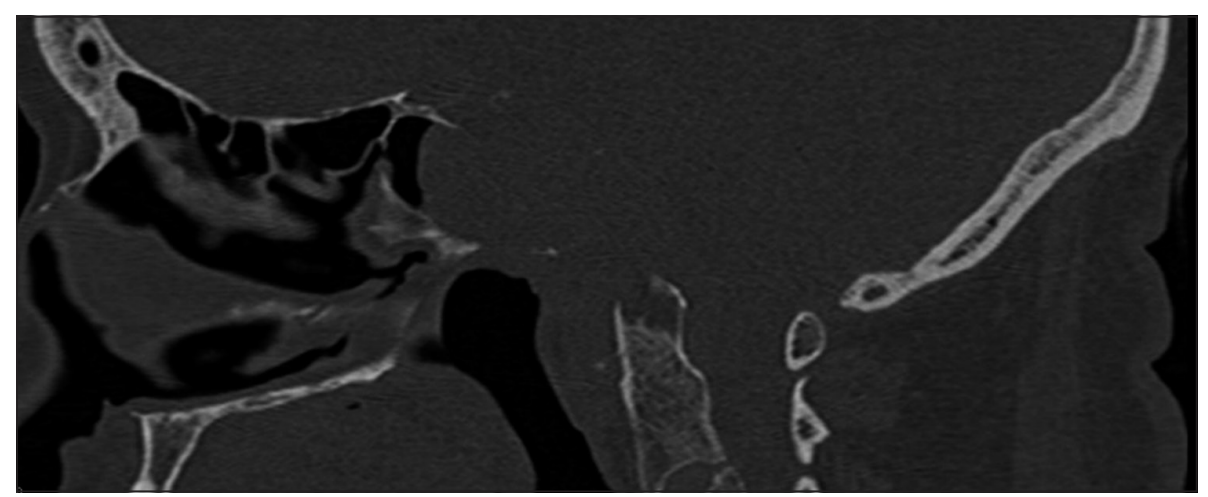




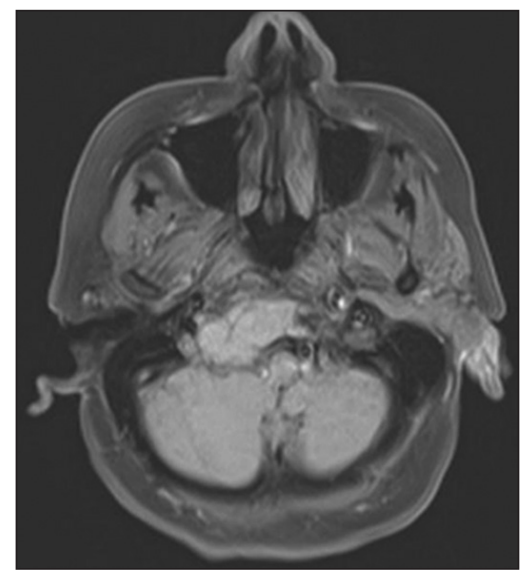

Figura 2. RMN T1 con contraste con lesión hiperintensa que ocupa fosa media y ángulo pontocerebeloso, hasta seno esfenoidal.

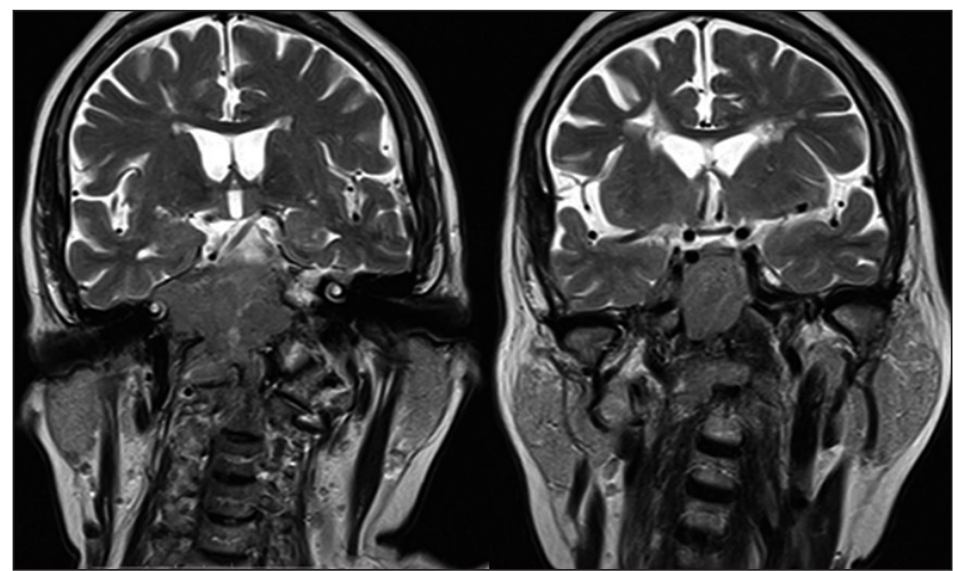

Figura 3. RMN T2 se observa una lesión isointensa que atraviesa línea media con erosión ósea e invasión a seno esfenoidal.

\section{Discusión}

Nuestra paciente se encuentra dentro del grupo etario más común para esta patología, mujer en la quinta década de la vida, sin embargo, su síntoma inicial y prácticamente único, fue el acúfeno, esto es diferente a lo reportado por la literatura. El dolor cervical y la rigidez presentada, no lo consideraba relevante al momento de la consulta. Los síntomas del plasmocitoma pueden variar de acuerdo del sitio afectado, siendo los más frecuentes: cefalea, déficit visual, parálisis de nervios craneales y anormalidades endocrinas ${ }^{4,5}$. De los 14 reportes de casos y series publicados (Tabla 1), el orden de frecuencia con que se presentan los síntomas iniciales son: déficit visual en 7 de los 14 casos, cefalea en 6/14, problemas cervicales $3 / 14$.

El acúfeno no está reportado en la bibliografía como un síntoma común, sin embargo, se encontró en 5/14 artículos publicados, de los cuales cuatro tenían carácter pulsátil. Los síntomas que se encontraron más frecuentemente fueron la cefalea, diplopía, la pérdida auditiva y las alteraciones cervicales ${ }^{1,2,4,5}$. En la resonancia magnética (RM) los plasmocitomas se observan como imágenes iso a hiperintensas en $\mathrm{T} 1$ con contraste, mientras que en T2 se observan imágenes iso a hipointensa, y en la tomografía de cráneo se aprecia una lesión destructiva. En el examen microscópico se observan pequeñas células con núcleo pequeño y cromatina atípica. La inmunohistoquímica expresa CD 138 en un $70 \%{ }^{6}$.

En una revisión de serie de casos publicado por Filippo Gagliardi y cols. en el año 2013 se incluyeron 25 casos, de los cuales solo 8 fueron considerados casos de plasmocitoma de clivus, con una edad media de 56,5 años, con predominio femenino 3:1 y los síntomas que se presentaron fueron cefaleas, síntomas endocrinológicos y déficit visual. Los autores no reportan la presencia de acúfeno ni hallazgos físicos como una lesión transmembrana. Los hallazgos característicos en RM fueron la hiperintensidad en imágenes ponderadas en T1, T2 y un fuerte realce por contraste después de administrar gadolinio. Se reveló una proliferación monoclonal de plasmocitos malignos con CD138, CD38 y CD56 positivos. La resección completa seguida de radioterapia fue el tratamiento de elección, solo el 85,7\% de los casos tuvieron una enfermedad estable después de un tiempo de seguimiento medio de 31,2 meses y solamente se informó un caso de muerte después de la progresión local ${ }^{7}$. Estos mismos autores ${ }^{7}$ presentaron 8 casos de su centro tratados con cirugía y radioterapia con progresión a mieloma múltiple en uno de los casos.

La inmunohistoquímica encontrada en los 14 reportes de casos y series de casos publicados demuestran que los marcadores tumorales 


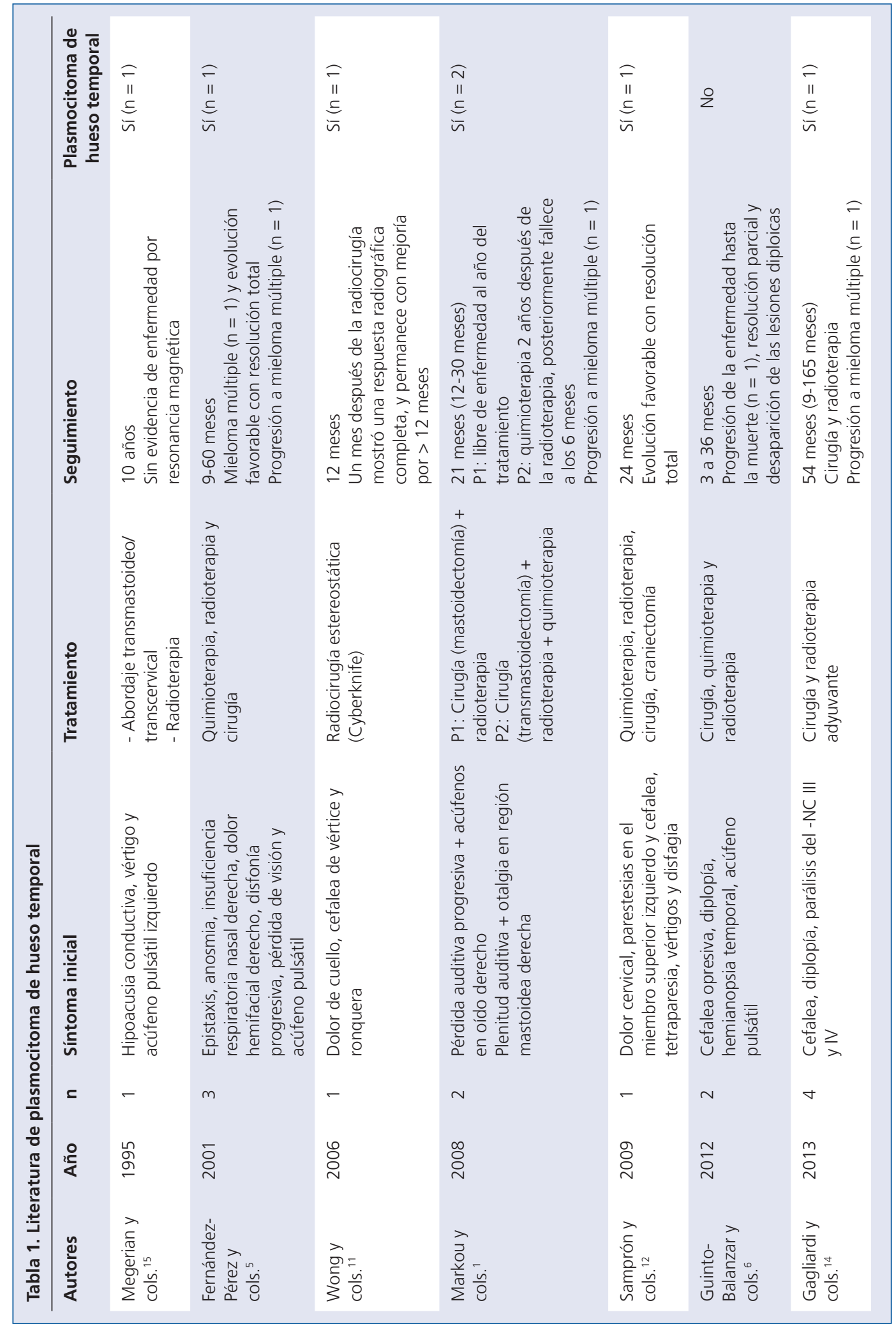




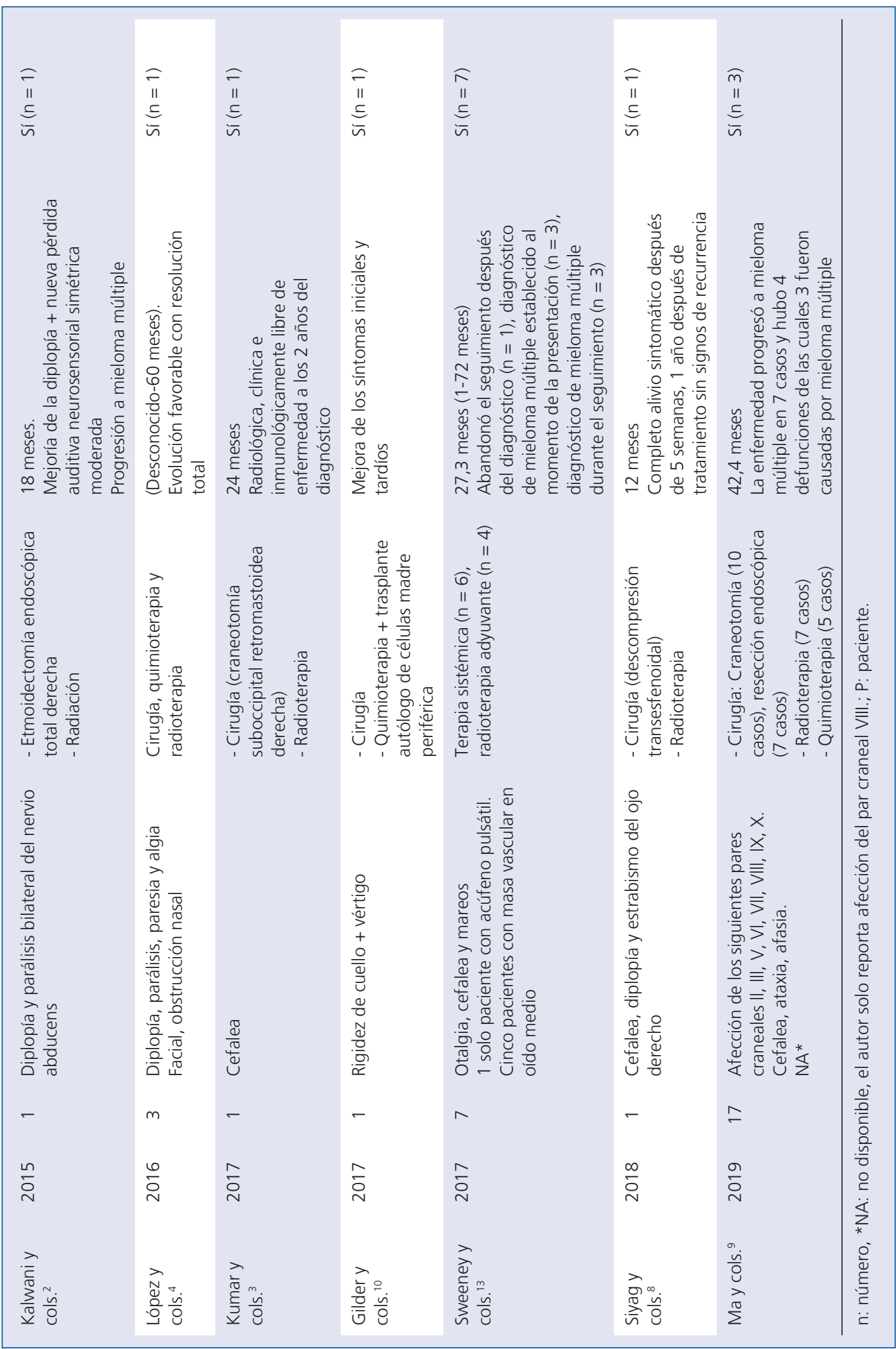


que se presentaron en un mayor porcentaje son: CD138 en 7 de los 13 reportes, CD38 y CD56 en 3/13, CD79 en 2/13 y CD128 en $1 / 13$, siendo el marcador tumoral más común el CD138 encontrado en un 70\% en la inmunohistoquímica del presente caso $^{2-9}$.

Los 45 pacientes publicados en los 14 reportes de casos y series de casos (Tabla 1) fueron tratados con radioterapia, excepto un paciente que se trató con cyberknife, sin embargo, varios pacientes con plasmocitoma de base de cráneo progresaron a mieloma múltiple, tratados con quimioterapia en la mayoría de las ocasiones.

El tratamiento recomendado es con radioterapia con una dosis de 50 Gy en un periodo de 4 semanas, aunque existen distintos protocolos reportados. Tres casos publicados por Fernández y cols. en 2001 fueron tratados con radioterapia con dosis de 45 Gy en 20 fracciones durante 4 semanas. Los resultados fueron variables, un paciente controlado, un paciente con progresión local que responde a quimioterapia y un paciente con progresión a mieloma múltiple ${ }^{5}$. Solo un paciente de los reportados en la literatura recibió trasplante autólogo de células madres periféricas ${ }^{10}$.

Un caso publicado por Eric T. Wong y cols. en el año 2006 experimentó dolor de cuello, cefalea de vértice y disfonía. La exploración neurológica mostró sensibilidad muscular en cuello, músculos paraespinales y supraespinoso; debido a la localización de la tumoración se trató con radiocirugía cyberknife. Un mes después del tratamiento mostró una resolución radiográfica completa ${ }^{11}$. El papel de la cirugía permite confirmar el diagnóstico, y puede ser útil en caso de descompresión. De los 14 reportes de casos y series de casos publicados ${ }^{1-6,8-15}$ (Tabla 1), varios fueron sometidos a cirugía de descompresión, cirugía de descompresión endoscópica y un paciente a cirugía con radiocirugía estereotáxica con cyberknife.

Afortunadamente, los plasmocitomas, son sensibles a radioterapia para un control local, la cirugía se reserva para diagnóstico y para descompresión paliativa. Se requiere un equipo multidisciplinario y seguimiento de por vida, debido a su alto riesgo a progresión a mieloma múltiple. Los médicos deben ser conscientes del riesgo de progresión a mieloma múltiple en plasmocitomas de base cráneo para su diagnóstico y tratamiento oportuno. La tasa de super- vivencia y vida sin enfermedad es del $74 \%$ y el $50 \%$ respectivamente, cuando hay recurrencia regularmente existe involucro sistémico, hasta 30 años posterior a su diagnóstico ${ }^{7}$. Debido a su baja incidencia, es difícil la realización de revisiones sistemáticas que permitan llegar a un consenso en cuanto al tratamiento óptimo del plasmocitoma de base de cráneo. Sólo existen escasos casos y series de casos publicados, los cuales se resumen en la Tabla $1^{1-6,8-15}$.

En una serie de casos de carácter multicéntrico en pacientes que se les diagnosticó plasmocitoma de hueso temporal entre los años 1990 y 2015, se describieron 7 casos, de los cuales 5 de ellos presentaron una masa vascular del oído medio de apariencia visible en la otoscopia. En las audiometrías 5 pacientes mostraron una hipoacusia conductiva asimétrica y 2 tuvieron una hipoacusia neurosensorial unilateral profunda ${ }^{13}$. Generalmente, la hipoacusia en estos casos es conductiva ${ }^{13,15}$, probablemente por la invasión a oído medio.

En la revisión de la literatura del presente artículo (Tabla 1), se identificaron 22 pacientes con involucro al hueso temporal (22 casos/45 totales), generalmente en la zona del ápex petroso. Es difícil conocer el sitio de origen del tumor. Lo más frecuentemente afectado en cabeza y cuello es el tejido submucoso de la nariz y senos paranasales, pero puede surgir de cualquier sitio. En el caso que presentamos se originó probablemente en el ápex petroso de lado derecho, y se extendió a través del esfenoides hasta $\mathrm{C} 3$ y el ápex petroso contralateral.

El acúfeno afecta la calidad de vida del paciente y puede ser síntoma de una enfermedad que ponga en riesgo la vida. Además, el abordaje diagnóstico en un paciente con acúfeno unilateral ya sea pulsátil o no pulsátil, es un reto para el médico. Es fundamental interrogar adecuadamente a nuestros pacientes e indicar los estudios de imagen de forma oportuna. El acúfeno es una causa común de consulta, sin embargo, es un signo de alarma que se presente de forma unilateral y todavía es más inusual que sea pulsátil, de ahí la importancia de que el otólogo, neurólogo y especialistas de la salud estén familiarizado y el amplio espectro de etiologías que pueden causarlo. En la revisión solo 5 estudios incluidos mencionan acúfeno como síntoma inicial ${ }^{1,5,6,13,15}$. Podría existir la posibilidad que el acúfeno no esté descrito 
en algunos estudios, pero sí se encuentre presente .

Megerian y cols. ${ }^{15}$ incluso comentan la posibilidad de que el plasmocitoma sea un diagnóstico diferencial de glomus yugular. Los autores presentan un caso de acúfeno pulsátil por plasmocitoma de hueso temporal (en el foramen yugular) y concluyen que una biopsia preoperatoria es importante en estos pacientes para normar conducta y diferenciarlo del glomus yugular. El acúfeno es una presentación inusual del plasmocitoma de hueso temporal, sin embargo, debe considerarse en pacientes con hipervascularidad del promontorio y otros datos neurológicos, como en este caso. Pueden presentarse, además, hipoacusia conductiva o neurosensorial, dependiendo de las estructuras que afecte el tumor.

\section{Conclusión}

El acúfeno es un síntoma común y una causa frecuente de consulta, con un amplio espectro de diagnósticos diferenciales. Sin embargo, puede ser el síntoma inicial de distintas patologías neurológicas incluyendo tumores, sobre todo aquellas localizadas en hueso temporal. El abordaje diagnóstico debe de estar guiado por el interrogatorio, la exploración física y estudios de imagen. Los plasmocitomas de base de cráneo son poco usuales así como su localización en hueso temporal, el acúfeno es un síntoma asociado a los mismos.

\section{Bibliografía}

1. Markou K, Karasmanis I, Goudakos J, Papaioannou M, Psifidis A, Vital V. Extramedullary plasmacytoma of temporal bone: report of 2 cases and review of literature. Am J Otolaryngol. 2009;30(5):360-365.

2. Kalwani N, Remenschneider A, Faquin W, Ferry J, Holbrook E. Plasmacytoma of the Clivus Presenting as Bilateral Sixth Nerve Palsy. J Neurol Surg Rep. 2015;76(01):156-159.

3. Kumar R, Kumar N, Mohindro S, Radotra B. Solitary plasmacytoma of temporal bone: A rare case report. Asian J Neurosurg. 2017;12(1):95.

4. López AO, Ortiz MM, Caballero GJ, Cruz PP, Camejo GY. Plasmocitoma solitario de la base craneal:

Reporte de tres pacientes. Rev Chil Neuropsiquiatr. 2016;54(1):34-40.

5. Fernández-Pérez A, Sancho-Mestre M, Gras-Albert J, Talavera-Sánchez J. Plasmocitoma solitario de cabeza y cuello. Presentación de tres casos y revisión de la literatura. Acta Otorrinolaringol Esp. 2001;52(8):715720 .

6. Guinto-Balanzar G, Abdo-Toro M, Aréchiga-Ramos N, Leal-Ortega R, Zepeda-Fernández E, NamboLucio MJ. Tumor de células plasmáticas del clivus: reporte de dos casos. Cir Cir. 2012;80.

7. Gagliardi F, Boari N, Mortini P. Solitary nonchordomatous lesions of the clival bone: differential diagnosis and current therapeutic strategies. Neurosurg Rev. 2013;36(4):513-522.

8. Siyag A, Soni T, Gupta A, Sharma L, Jakhotia N, Sharma S. Plasmacytoma of the Skull-base: A Rare Tumor. Cureus. 2018;10(1).

9. Ma X, Li D, Wang L, Hao S, Zhang L, Zhang J et al. Clinical features, radiological profiles, and surgical outcomes of primary intracranial solitary plasmacytomas: a report of 17 cases and a pooled analysis of individual patient data. J Neurooncol. 2019;142(2):263-272.

10. Gilder H, Murphy M, Alvi M, Kerezoudis P, Shepherd D, Maloney P, et al. Skull base plasmacytoma: A unique case of POEMS syndrome with a plasmacytoma causing craniocervical instability. $J$ Clin Neurosci. 2018;47:254-257.

11. Wong E, Lu X, Devulapalli J, Mahadevan A. Cyberknife Radiosurgery for Basal Skull Plasmacytoma. J Neuroimaging. 2006;16(4):361-363.

12. Samprón N, Arrazola M, Urculo E. Plasmocitoma de base craneal con inestabilidad cráneo-cervical. Neurocirugía. 2009;20(5):478-483.

13. Sweeney A, Hunter J, Rajkumar S, Lane J, Jevremovic D, Carlson M. Plasmacytoma of the Temporal Bone, a Great Imitator. Otol Neurotol. 2017;38(3):400-407.

14. Gagliardi F, Losa M, Boari N, Spina A, Reni M, Terreni MR, Mortini P. Solitary clival plasmocytomas: misleading clinical and radiological features of a rare pathology with a specific biological behaviour. Acta Neurochir. 2013;155(10):1849-56.

15. Megerian CA, McKenna MJ, Nadol Jr JB. Nonparaganglioma jugular foramen lesions masquerading as glomus jugulare tumors. Otol Neurotol. 1995;16(1):94-98. 